(a)

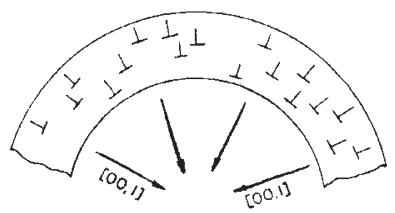

(b)

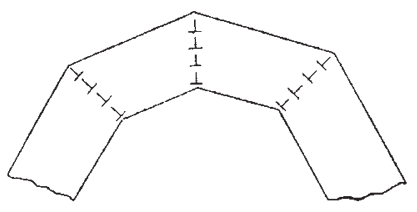

Fig. 4

in Fig. $4 b$, on a greatly exaggerated scale. From the degree of polygonization we estimate on the average an edge dislocation about every 200 atoms along the boundary.

We are indebted to Dr. W. W. Piper of this laboratory for making available to us these crystals, which he has grown by a vapour-phase technique, and to D. Hallgren for assistance in the measurements.

Research Laboratory,

W. L. Roth

General Electric Company,

Schenectady, New York.

Aug. 5.

\section{A New Method of transporting Optical Images without Aberrations}

'l'He transportation of optical images has been carried out hitherto with the aid of lenses or mirrors or both. As with all optical systems, aberrations are introduced and the parts have to be aligned carefully; it seemed worth while, therefore, to search for a method by which no aberrations are introduced and which allows (strong) deviations from alignment without deterioration of the image. Consideration of the construction of the eye of some insects suggested another approach. If a bundle or sheaf of thin transparent fibres is cut off perpendicularly at both ends and an optical image is formed on one end, it will be seen at the other end, as the light entering one fibre can only leave this at the other end, provided leakage of light from one fibre to another of the bundle is prevented. Moreover, the cylindrical wall of each fibre must reflect the light as nearly completely as possible, because of the very numerous reflexions occurring when the fibres are thin compared to their length. Preliminary experiments, started in January 1950, have shown that coating the fibres with silver or any other metal yields an unsatisfactory transmission. A much better result was obtained when the fibres were coated with a layer of lower refractive index, which ensured total reflexion. This coating was isolated from the neighbouring fibres by a thin coat of black paint. In this way, flexible 'image rods' have been obtained with satisfactory transmission, a very good contrast in the end image, and with the possibility of using forms bent in any direction (up to at least $360^{\circ}$ ).

The first models were made of glass fibres. Much better transmission was obtained by means of plastic fibres, coated with either plastic of low refractive index or other transparent material of low refractive index. With an index of 1.52 for the core and 1.47 for the coating, the effective angular aperture of the light pencils entering and leaving the 'image rod' appeared to be about $1: 1 \cdot 8$, which is ample for most practical applications, though a smaller difference of refractive indices would have been sufficient theoretically. Transmission, of course, is highly dependent on the transparency of the material used.

In order to obtain a high resolving power, the diameter of the fibres (or tubes) must be small. It appeared practicable to go down to $0.1 \mathrm{~mm}$. for the core, though it seems possible to utilize smaller diameters. With the smaller diameters diffraction will play a preponderant part, and the fibres are then 'wave guides' for visible light. Of course, resolving power and overall transmission are reduced by the thickness of the coatings. The low-index coating must have at least a thickness of three to five times the wave-length. The length of the samples prepared in this laboratory varies from 6 to $20 \mathrm{~cm}$.

Two obvious applications may be mentioned: cystoscopes and apparatus for the coding of twodimensional pictures. Coding and decoding of twodimensional pictures proved to be practicable.

The apparatus is different from the compound eye of an insect in that with the latter each 'fibre' has its own entrance lens, while with 'image rods' an image is formed on the entrance end by means of a system outside of the rod. Of course, entrance and exit surfaces of the rod may have another form than plane, for example, spherical.

This work was done under contract with the National Defence Research Council.

\section{A. C. S. van Heel}

Laboratorium voor Technische Physica,

Delft.

May 21.

\section{A Flexible Fibrescope, using Static Scanning}

As optical unit has been devised which will convey optical images along a flexible axis. The unit comprises a bundle of fibres of glass, or other transparent material, and it therefore appears appropriate to introduce the term 'fibrescope' to denote it. An obvious use of the unit is to replace the train of lenses employed in conventional endoscopes. The existing instruments of this kind, for example, cystoscopes, gastroscopes and bronchoscopes, etc., consist of a train of copying lenses and intermediate field lenses. They are either rigid or have only limited flexibility. Moreover, the image quality of these systems is poor, since they consist only of positive lenses which give rise to a very large curvature of field. In existing gastroscopes the total number of lenses employed may be as many as fifty, and in consequence the light transmission is poor, due to the total glass path and the number of air-glass surfaces, in spite of blooming. Even more important in this respect, however, is the need to use small relative apertures for such instruments, this being necessary if acceptable definition is to be obtained with such large field curvature.

What was thought to be an entirely new approach to the problem of conveying images along a flexible axis was proposed by one of us (H. H. H.) as long 